\title{
Effect of the environmental conditions on the ultimate load of damaged unidirectional of natural hemp fiber/epoxy composite
}

\author{
M.A. Khiat ${ }^{1}$, R. Zenasni ${ }^{1, a}$, Z. Serier ${ }^{2}$ and Jaime Vina Aurelio Olay ${ }^{3}$ \\ 1 University of Mostaganem, Dept. of Mechanics, Algeria \\ 2 University of Sciences and Technology of Oran, Laboratory of composite structures and innovative materials, Algeria \\ 3 University of Oviedo, Dept. of material Sciences, Campus de Viesques, 33204 Gijon, Spain
}

Received 16 March 2015, Accepted 19 June 2015

\begin{abstract}
Natural continuous natural fibres (hemp, flax, etc.) are gaining popularity in composite materials because they can advantageously replace glass fibers. It is found that intrinsic properties of unidirectional (UD) composites are almost equivalent to those of unidirectional glass fiber composites. Unfortunately it is difficult to get repeatable results because of the inherent variability in the properties of natural fibers compared to glass fibers. Their quality is largely affected by the weather conditions, the extraction location along the plant and the techniques used to extract them (retting, bleaching, etc.). The present paper proposes a strength reliability model for unidirectional composites with natural fibers in a hexagonal array. The model assumes that, a central core of broken fibers flanked by unbroken fibers which are subject to stress concentrations from the broken natural fibers. Thermal and hygroscopic residual stresses are neglected because they haven't more effect when the composite was subjected to tensile than transverse loading. The approach of the model consists of using a modified shear lag model to calculate the ineffective lengths and stress concentrations around the broken fibers. In this paper, we attempt to incorporate in the proposed model the unidirectional composite property variation with temperature and moisture in order to predict even composite strength degradation. Strength degradation is often seen as a result of changes in ineffective lengths at natural fiber breaks and the corresponding stress concentrations in intact neighboring fibers.
\end{abstract}

Key words: Unidirectional composite / fracture / stress concentrations / temperature / humidity / aging

\section{Introduction}

Natural fiber reinforced composites are actively under investigation considering their potential to reduce or eliminate some of the problems associated with the nondegradable glass and carbon fibers in composites. Natural fibers are fully biodegradable, they consume globally less energy for their production, and they maintain acceptable specific stiffness, strength and toughness compared to glass fibers. They are already used as fiber mats or fabrics for the manufacturing of a few automotive interior parts. Prototypes of a roof structure and I-beams have also been developed successfully. These materials allow sufficient stiffness and strength for nonstructural interior panels but in the case of mats, the fiber volume content that can be achieved is limited, especially when it is used to mold parts with the resin infusion process (this limit is currently imposed by the use of a vacuum bag in combination with only the atmospheric pressure as the driv-

\footnotetext{
${ }^{a}$ Corresponding author: zramdane@netcourrier.com
}

ing pressure). In this case, the mechanical properties are much more influenced (or driven) by the matrix than in the case of unidirectional composites. On the other hand mats are usually considered as high permeability reinforcements compared to unidirectional reinforcements or fabrics and are thus easier to impregnate. For the fabric alternative (stitched or woven), it seems that the minimum level of twist of the yarns required to allow proper fabrication and the weave pattern obtained in these architectures are such that the yarn permeability and the tensile properties (mainly the modulus and strength along the fiber direction) are highly reduced. For applications requiring higher strengths and stiffnesses, it thus seems logical to use natural fiber composites made of unidirectional plies with well oriented fibers such as what is usually obtained with UD prepregs made of synthetic glass or carbon fibers. Indeed and up to now, it seems that for natural fibers the highest mechanical properties were obtained along the fiber direction with the fibers laid down as straight as possible. 
The composites give an additional liberty for a designer to choose the material behaviour $[1,2]$ according to the type of fibers and resin, the rate of the reinforcement, the fiber orientations and the process of manufacturing. Moreover, the choose of the optimal material, allowed by the use of the composites, is counterbalanced by the greatest variability of certain mechanical parameters, in particular the ultimate fracture strength.

Two of these properties are essential in the dimensioning of composite structures, it is about the micromechanical and macromechanical characterization scale of the elastic modulus and the mechanical resistance. A synthesis of these properties can be found in work of Christensen [3]. This work is based on the micromechanical homogenization models using a representative elementary volume. Many researches have been conducted, the models of Zweben and Rosen [4,5] which considered among the oldest models for the prediction of the polymeric composite resistance. The two authors studied the damage of a structured fiber arrangement and where they determinated the effective length to estimate the tensile strength, while basing on the fiber shearing analysis oriented in unidirectional direction. However their models do not take in account the effects of the stress concentrations in the adjacent fibers to those of broken fibers. Phoenixes et al. [6] have determinated the stress and the statistical lifetime failure of the unidirectional carbon fiber. Their model consists of seven parallel carbon fibers forming an hexagonal arrangement in an epoxy matrix. In the same context, Landis et al. [7] investigate the problem of how to choose the effective dimensions of long fibers composite by mean of a finite element method. Their model also takes in account the direct interactions of broken fibers with the intact most nearest fibers. Thereafter, the Landis model [7] was improved by Landis and Mc Meeking [8] by taking into account the effects of the axial slip to the fiber/matrix interface and the locating of the intact fibers on the stiffness by evaluating the stress concentration around the broken fiber. Case et al. [9] proposed a technique of general analysis completely different from the preceding models, for the study of the unidirectional composite material behaviour containing broken fibers. The model is based on the fiber spacing and that to have a near representation to the reality of neighbour intact fibers. The succession of the failure fibers was modelled by the fiber actualization method. Case and Reifsnider [10] also studied the problem of a central crack in several concentric cylinders. The problem was solved by applying the assumptions of standard elasticity, with a suitable choice of the stress functions for each component. This solution was applied to the problem of fibers failure in the unidirectional composite material by making geometrical assumptions. Foster [11] proposed a direct numerical simulation and an analytical model to predict the resistance of the composites in tensile traction and flexion. By using a regular square arrangement of intact fiber, he determined the resistance of a $\mathrm{Ti}$ matrix $6 \mathrm{Al}-4 \mathrm{~V}$ reinforced by $\mathrm{SiC}$ fibers. He also demonstrate that the fibers failure occurs in a random way when the load reaches the composite limit strength. Consequently, the statistical accumulation of this failure can lead to the total damage of the composite. Gao and Reifsnider [12] proposed an analysis of the distribution load using the Batdorf probabilistic model to predict the tensile strength. By using the assumptions of the delayed shearing action "shear-lag", they studied the failure caused by the shearing at the fiber/matrix interface by defining a shearing parameter used to describe the complete elastic behaviour of the fiber/matrix with a perfectly plastic matrix debonding.

During the last years, the use of natural composites fibers involved a great interest, particularly in the area of organic composites matrix [13]. Because of a great increasing demand of ecological materials and increasingly high cost of the synthetic fibers (carbon or glass), new materials containing natural fiber were developed. They have the advantage of being lighter while having interesting mechanical properties [13]. Moreover, the natural fibers have a much less cost than the synthetic and they are recyclable. The recent advances in the use of natural fibers (flax, cellulose, bamboo, hemp, sisal...) in the composites were studied by several authors [14-16], they reported that the conventional fibers can be produced with reproducible characteristics, contrary to the vegetable fibers which are depended on their diameter, of their structure, of their degree of polymerization or of their origin in the plant (peripheral part or intern in the stem). The chemical composition of natural fibers also depends on the season when they were sown, collected, or of the type of the ground.

The comparative study of the life cycle of automotive part or other made in epoxy matrix reinforced by hemp fibers, and a part manufactured from polystyrenebutadiene acrylonitrile (ABS) according to various methods, revealed that the part reinforced with hemp fibers present an advantage from the environment view point during the phase of manufacturing $[17,18]$. Finally, the use of natural hemp fibers as reinforcement can allow to reach interesting mechanical properties, while reducing the composite costs.

An analysis of the process of longitudinal failure including the micromechanical interactions between natural fibers and the matrix is carried out by taking in account the effect of the environmental conditions on the degradation of the composite components. This model is based on Gao and Reifsnider [12] which allow to predict the resistance and the durability of the unidirectional composite by using micromechanical techniques. The main purpose of the present study is to exploit the hemp natural fibers due to the increase demand and a low cost than the synthetic fibers while having the interesting mechanical properties. The modified Gao and Reifsnider model was used to predict the tensile behaviour of hemp fiber/epoxy composite subjected to moisture and temperature aging.

\section{Model of longitudinal fracture}

The hygrothermal behaviour of the laminated hemp/epoxy composites is extensively determined by the 
Table 1. Temperature and humidity parameter variations [12].

\begin{tabular}{ccccc}
\hline$T_{g}^{0}\left({ }^{\circ} \mathrm{C}\right)$ & $T_{\mathrm{rm}}\left({ }^{\circ} \mathrm{C}\right)$ & $g\left({ }^{\circ} \mathrm{c} / \mathrm{c}\right)$ & $a$ & $f$ \\
\hline 160 & 22 & 2000 & 0.5 & 0.04 \\
\hline
\end{tabular}

matrix properties to the interfaces, on the other hand the natural hemp fibers are relatively sensitive to the increase in moisture and temperature. The effect of moisture generates residual stresses, the plasticity of polymers as well as degradation at the interfaces [19,20]. Moreover, the plasticity can involve the reduction in glass transition temperature [21], which can affect the composite behaviour in high temperature environments. To introduce the temperature effect and the variation of moisture concentration on the mechanical properties, Tsai [21] proposed the non-dimensional temperature $T^{*}$, which is the principal parameter to evaluate the hygrothermal characteristics of the composite.

$$
T^{*}=\frac{T_{g}-T_{\mathrm{opr}}}{T_{g}-T_{\mathrm{rm}}}
$$

If $T_{g}$ is the glass transition temperature of the matrix, Topr is the operation temperature and $T_{\mathrm{rm}}$ is the room reference temperature. It is also supposed that the moisture removes the glass transition temperature in a dry environment $T_{g}^{0}$ by a change of relative temperature for unit absorbed moisture " $g$ ",

$$
T_{g}=T_{g}^{0}-g C
$$

We can use the non dimensional temperature $T^{*}$ in an empirical way to express the matrix and the fiber rigidities $\left(E_{m}, E_{f}\right)$ in the way:

$$
\begin{aligned}
& E_{m}=E_{m}^{0}\left(T^{*}\right)^{a} \\
& \frac{E_{f}}{E_{f}^{0}}=\frac{G_{f}}{G_{f}^{0}}=\left(T^{*}\right)^{f}
\end{aligned}
$$

The index " 0 " indicates the initial values of the matrix and the fibers rigidities respectively, $a$ and $f$ represent the hygrothermal characteristics parameters given in Table 1.

We used the model of Gao and Reifsnider [12] to estimate the strength of unidirectional composite. This model allows us to introduce the variation of the mechanical characteristics of the matrix and natural fibers as function of the temperature and the moisture in order to predict the unidirectional composite strength. This strength varies generally in terms of changes of the ineffective length $\delta$ in the zone of the fiber failure and the stress concentrations corresponding to the intact neighbouring fibers. The ineffective length is generally defined as the length taken between the fiber fracture zone until the length for which the fiber renews its capacity to bear the complete loading. This concept is illustrated in Figure 1, where $\sigma_{f}$ is the fiber stress. The changes of the ineffective lengths can be as function of the temperature and the moisture due to the change of the matrix and of

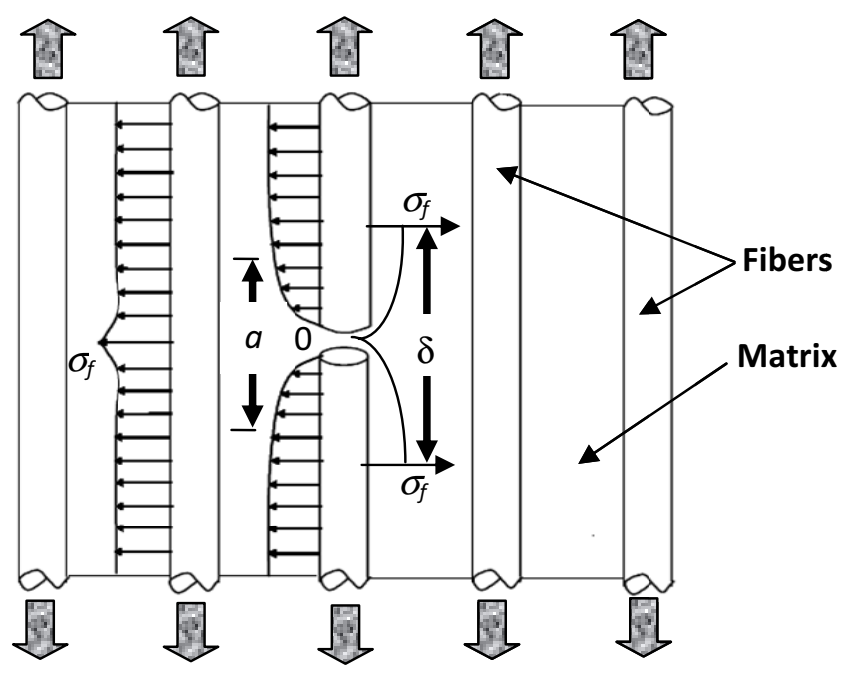

Fig. 1. Broken fibers with the elongation of the ineffective length.

the fibers properties. The model approach consists in employing the shear stress condition at the interface in order to calculate the ineffective lengths and the stress concentration around the broken natural fibers. Thermal and hygroscopic residual stresses generally increase with the increase of fiber volume and have more effect for transverse loading than tensile loading. when starting the fiber break usually the residual stresses can be neglected.

According to Gao and Reifsnider [12], the tensile fiber reinforced composite properties, depend on the strength, the elastic fiber modulus, the strength and the matrix chemical stability and also of the adherence effectiveness to the interface fiber/matrix during the load transfer. In the case of the hemps fibers and even as carbon fibers which we used in the preceding study, the elastic modulus and the fiber mechanical resistance remain almost unchangeable with the variation in the temperature and the moisture, contrary, the epoxy matrix receives changes of the properties. These changes are used in this study; however, because of the difficulty detected during the determination of the properties at the interfaces. The adherence resistance at the interfaces is considered as being related to the matrix yield stress and the shear interface $\tau_{0}$. The crack at the interface will take place when the interface shear stress reached $\tau_{0}$.

The model of Gao and Reifsnider [12] is distinguished from the other models by the presence of the effect of shearing and it takes in account the local damages in the form of debonding at the interface and plasticity. In this area, the matrix and the interface shear stress was considered as constant with a value $\eta \tau_{0} . \eta$ is a shear parameter defining the shear stress in the inelastic area. When $\eta$ tends to zero, no transfer of shearing took place between the broken fibers and their neighbours in this area. It would be the case in the area completely detached, or from the cracked matrix. Contrary when $\eta=1$, the shear stress in the inelastic area is equal to $\tau_{0}$. For the application of this model in this study, we used $\eta=1$. 
A representative diagram of this problem is given in Figure 2. This model supposes that exist a central cell of i broken fibers which are surrounded by unbroken fibers exposed to stress concentrations due to broken fibers [22]. The unbroken fibers are in their turn surrounded by an effective homogeneous material which deformed uniformly.

We suppose that the broken cell can be assimilated to an homogeneous material with a transversal circular cross section whose Young modulus can be obtained by the rule of mixtures:

$$
E=\frac{i A_{f} E_{f}+\left[i A_{m}-\pi\left(r_{0}^{2}-\left(r_{0}-d\right)^{2}\right)\right] E_{m}}{\pi\left(r_{0}-d\right)^{2}}
$$

where $A$ corresponds to the area and $E$ corresponds to the equivalent Young modulus. The substituted properties with $f$ refer to the fiber properties, while those substituted with $m$ refer to the matrix properties. The parameters $A_{f}, A_{m}$, radius $r_{0}$ and the diameter $d$ are given by the following equations:

$$
\begin{aligned}
A_{f} & =\pi r_{f}^{2} \\
A_{m} & =\pi\left(r_{f}+d\right)^{2}-\pi r_{f}^{2} \\
r_{0} & =\sqrt{i\left(r_{f}+d\right)^{2}} \\
d & =\frac{r_{f}}{\sqrt{V_{f}}}-r_{f}
\end{aligned}
$$

Local damages are included in the model by introducing of debonding area and local plasticity (Fig. 2). The matrix shear stress and the interface are considered as constant for the value $\eta \tau_{0}$, the equilibrium equations in this zone $(0 \leq x \leq a)$ are:

$$
i A_{f} E_{f} \beta \frac{d^{2} U_{0}}{d x^{2}}-2 \pi r_{0} \eta \tau_{0}=0
$$

$i A_{f} E_{f} \frac{d^{2} U_{1}}{d x^{2}}+2 \pi\left(r_{0}+2 d+2 r_{f}\right) \frac{G_{m}}{2 d}\left(U_{2}-U_{1}\right)+2 \pi r_{0} \eta \tau_{0}=0$

$\beta$ was given as function of the fiber and the matrix geometrical modulus respectively, $a$ represents the half length of the debonding area locally plasticized.

$U_{0}, U_{1}$ and $U_{2}$ represent the stresses and the displacements in the three zones (see Fig. 2).

The matrix shear modulus was given by:

$$
G_{m}=\frac{E_{m}}{2\left(1+\nu_{m}\right)}
$$

The solution of Equations (10) and (11) with the boundary conditions was:

For $x=0$, the stress $\sigma$ in the broken core was equal to 0 , thus $\frac{\mathrm{d} U_{0}}{\mathrm{~d} x}=0$ while for $x=0$, the displacement $U_{1}=0$ (symmetry).

By considering that the neighboring fibers of the broken core are not affected by the broken core, (i.e $U_{2}=$

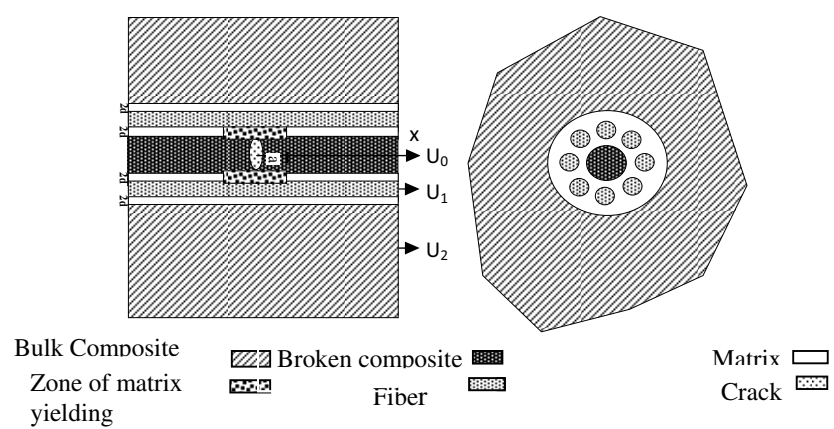

Fig. 2. Unidirectional composite with transverse fiber fracture and local damage of matrix and adjacent fibers.

$\frac{\sigma_{c}}{E_{c}} x$, where $c$ was the entire composite index), we can obtain a solution for $U_{0}$ and $U_{1}$. After introducing the necessary conditions, the Equations (10) and (11) are written in the form:

$$
\begin{aligned}
U_{0}(x)= & \frac{\eta \tau_{0}}{r_{0} E_{1}} x^{2}+C_{0} \\
U_{1}(x)= & \mathrm{C}_{1}\left(e^{\lambda_{1} x}-e^{-\lambda_{1} x}\right)+\frac{2 r_{0} \eta \tau_{0}}{\left(R_{2}^{2}-r_{0}^{2}\right) E} \frac{1}{\lambda_{1}^{2}}\left(1-e^{-\lambda_{1} x}\right) \\
& +\frac{\sigma_{C}}{E_{C}} x
\end{aligned}
$$

where $C_{0}$ and $C_{1}$ are the integration constants and, $R_{2}$ was the geometrical coefficient given by:

$$
R_{2}=r_{0}+2 r_{f}+d \quad \text { and } \quad \lambda=\lambda_{1}=\sqrt{\left(\frac{1}{R_{2}^{2}-r_{0}^{2}}\right) \frac{G_{m}}{E_{c}} \frac{1}{2 d}}
$$

$E_{1}$ was the adjacent intact fiber modulus given by:

$$
E_{1}=\frac{E_{f} A_{f}+E_{m} A_{m}}{A_{f}+A_{m}}
$$

Under the tensile load $\sigma_{c}$, the entire composite modulus $E_{c}$ was given by the rule of mixtures:

$$
E_{c}=E_{f} V_{f}+\left(1-V_{f}\right) E_{m}
$$

Also for the zone $a \leq x \leq \infty$, where there is no yield at the interface, the equilibrium equations are:

$$
\begin{gathered}
E \pi\left(r_{0}-d\right)^{2} \frac{d^{2} U_{0}}{d x^{2}}+2 \pi r_{0} \frac{G_{m}}{2 d}\left(U_{1}-U_{0}\right)=0 \\
n_{i} A_{f} E_{f} \frac{d^{2} U_{1}}{d x^{2}}+2 \pi\left(r_{0}+2 d+2 r_{f}\right) \frac{G_{m}}{2 d}\left(U_{2}-U_{1}\right) \\
-2 \pi r_{0} \frac{G_{m}}{2 d}\left(U_{1}-U_{0}\right)=0
\end{gathered}
$$

where $n_{i}$ represents the number of the adjacent broken fibers. To satisfy the equations, the expression of the displacements are given as below:

$$
\left.\begin{array}{l}
U_{0}=\bar{U}_{0} e^{-\gamma x} \\
U_{1}=\bar{U}_{1} e^{-\gamma x} \\
U_{2}=\frac{\sigma_{C}}{E_{C}} x
\end{array}\right\}
$$


By substituting the Equation (19) in (17) and (18), we obtain the differential equations:

$$
\begin{aligned}
U_{0}(x)= & C_{2} e^{-\sqrt{\gamma_{1}} x}+C_{3} e^{-\sqrt{\gamma_{2}} x}+\frac{\sigma_{c}}{E c} x \\
U_{1}(x)= & C_{2}\left(1-\frac{\gamma_{1}}{A}\right) e^{-\sqrt{\gamma_{1}} x}+C_{3}\left(1-\frac{\gamma_{1}}{A}\right) e^{-\sqrt{\gamma_{2}} x} \\
& +\frac{\sigma_{c}}{E_{c}} x
\end{aligned}
$$

where: $\gamma_{1}, \gamma_{2}, A, B$ and $D$ are the algebraic equations:

$$
\begin{aligned}
\gamma_{1} & =\frac{(A+B+D)-\sqrt{(-A-B-D)^{2}-4 A B}}{2} \\
\gamma_{1} & =\frac{(A+B+D)+\sqrt{(-A-B-D)^{2}-4 A B}}{2} \\
A & =\frac{G_{m}}{r_{0} E_{1} d} ; B=\frac{r_{0} G_{m}}{\left(R_{2}^{2}-r_{0}^{2}\right) E_{2} d} ; D=\frac{R_{2} G_{m}}{\left(R_{2}^{2}-r_{0}^{2}\right) E_{2} d}
\end{aligned}
$$

where: $C_{2}$ and $C_{3}$ are the integration constants calculated at the continuity conditions for $x=a$ :

$$
\begin{aligned}
& {\frac{\mathrm{d} U_{0}}{\mathrm{~d} x}}_{(0 \leq x \leq a)}=\frac{\mathrm{d} U_{0}}{\mathrm{~d} x}{ }_{(a \leq x \leq \infty)} \\
& {\frac{\mathrm{d} U_{1}}{\mathrm{~d} x}}_{(0 \leq x \leq a)}=\frac{\mathrm{d} U_{1}}{\mathrm{~d} x}{ }_{(a \leq x \leq \infty)}
\end{aligned}
$$

The length $a$ can be calculated by imposing the shear stress continuity condition at $x=a$. We know that the shear stress $\tau_{0}$ is considered as constant in the zone $0 \leq$ $x \leq a$ (zone of plasticity), at the outside plasticized zone $a \leq x \leq \infty$, the stress was given by:

$$
\tau(x)=\left(U_{0}(x)-U_{1}(x)\right) \frac{G_{m}}{2 d}
$$

The shear stress continuity is satisfied at $x=a$ if:

$$
\tau_{0}=\left(U_{0}(a)-U_{1}(a)\right) \frac{G_{m}}{2 d}
$$

When the value of $a$ is calculated, we can obtain the $U_{0}(x)$ and $U_{1}(x)$ as function of the known quantities. It should be noted that the solutions must be found for each possible number of adjacent fractures, and for each considered load step. The stress concentrations $C_{i}$ for $i$ brocken fibers, are:

$$
C_{i}=\frac{\left.\frac{\mathrm{d} U_{1}(x)}{\mathrm{d} x}\right|_{x=0}}{\sigma_{c} / E_{c}}
$$

The ineffective lengths can be calculated by solving for the distances along " $x$ " taken for the stresses along the broken fibers to reach the applied stress value (where $C_{i}=1$ ). For $n$ progressive loading, the tensile stress $\sigma_{c}$ was a fraction of the fibers strength $\sigma_{0}$. If $j$ was the increase in load, we can write:

$$
\sigma_{c}=\frac{\sigma_{0} j}{n}
$$

The ineffective length $\delta$ is a required parameter for the different fracture models. The length $\delta$ was determinated from some experimental measures for a crack configuration by mean of spectroscopy [23]. However, the systematic renewal of measure for different types of composites would be costly. For this reason, we used some estimable models of $\delta[4,24]$. For an hexagonal arrangement, the expression of $\delta$ is given by :

$$
\delta=\frac{1}{2}\left[\ln \left(\frac{\pi}{6 V_{f}}\right) \frac{E_{f} A_{f}}{2 \pi G_{m}}\right]^{\frac{1}{2}} 2 r_{f}
$$

\section{Model validation}

First, we carried out many applications on plate of graphite/epoxy laminate. The mechanical and the geometrical characteristics are given respectively in Table $2[15,24,25]$. To appreciate the damage evolution at the zones locally plasticized; we carried out a progressive increase in the external load consequently an increase of the applied tensile stress applied to the sample edges. The increase in the load, has enabled us to quantify the elongation of the zone locally plasticized and the ineffective zone for which the fiber renewal with its capacity to bear the complete loading and that according to the number of broken fibers. Finally, we will determine the evolution of longitudinal displacement of broken and intact fibers according to the sample length.

The validation of our model was carried out by comparison with the Foster analysis method [11] for a square arrangement of unidirectional broken fibers and that for an hexagonal arrangement graphite/epoxy composite [18]. From Figure 3, the stress concentration increases according to the number of broken fibers. The maximum difference between the Foster curves [11] and the results [18] is lower than 5\%. This difference is due to the choice of the fiber arrangement which is hexagonal for the hemp/epoxy composite [18] and square arrangement in Foster models. On the other hand in model of hemp/epoxy composite and that of graphite/epoxy, the maximum difference was lower than $7 \%$ due to the fiber arrangement and the fiber nature under the effect of the environmental conditions. We can conclude that our results are in good agreement with those of Foster [11] and the preceding results [18].

\section{Hygrothermal analysis}

The hygrothermal behavior of the laminated hemp/epoxy composite is largely determined by the matrix properties at the interfaces, on the other hand the natural fibers are relatively sensitive to the increase in moisture and temperature. Many authors [19,20] noticed that the effect of moisture generates the sensitivity of the composites to residual stresses, polymer plasticization as well as the degradation at the interfaces. Moreover, the plasticity can involve the decrease in glass transition temperature [21], which can affect the composite behavior at high temperature environments. 
Table 2. Mechanical and geometrical characteristics of hemp/epoxy composite $[15,24,25]$.

\begin{tabular}{lc}
\hline Fiber Young modulus $E_{f}$ & $27.6 \mathrm{GPa}$ \\
Matrix Young Modulus $E_{m}$ & $4.2 \mathrm{GPa}$ \\
Fiber reference resistance $\sigma_{0}$ & $3.10 \mathrm{GPa}$ \\
Shear stress $\tau_{0}$ & $25.20 \mathrm{MPa}$ \\
Matrix Poisson coefficient $\nu_{12 m}$ & 0.43 \\
Fiber volume fraction $V_{f}$ & 0.51 \\
Shear parameter $\eta$ & 1.0 \\
Specimen length $L$ & $200 \mathrm{~mm}$ \\
Specimen reference length $L_{0}$ & $20 \mathrm{~mm}$ \\
Specimen width $W$ & $10 \mathrm{~mm}$ \\
Thickness specimen $t$ & $8 \mathrm{~mm}$ \\
Fiber radius $r_{f}$ & $120 \mu \mathrm{m}$ \\
Maximal number of broken fibers $n_{i}$ & 40 \\
Matrix thermal coefficient expansion $\alpha m$ & $63 \times 10^{-6} \mathrm{~m} / \mathrm{m} /{ }^{\circ} \mathrm{C}$ \\
Matrix hygroscopic coefficient $\beta m$ & $0.33 \mathrm{~m} / \mathrm{m} / \mathrm{kg} / \mathrm{kg}$ \\
Hemp fiber thermal coefficient expansion $\alpha f$ & $5 \times 10^{-6} \mathrm{~m} / \mathrm{m} /{ }^{\circ} \mathrm{C}$ \\
Hemp fiber hygroscopic coefficient $\beta f(\%)$ & 8 \\
Hemp density (g/cm ${ }^{3}$ ) & 1.48 \\
Elongation at failure $(\%)$ & 47 \\
\hline
\end{tabular}

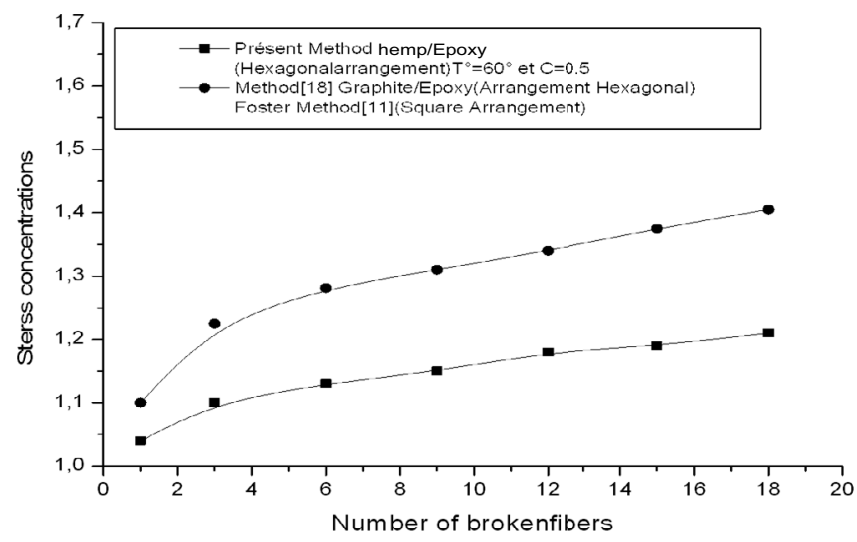

Fig. 3. Comparison of the stress concentrations for normal environmental conditions, models validations $[11,18]$.

\subsection{Evolution of ineffective lengths}

The ineffective length $\delta$ is defined as being the length ranging between the fractured zone fibers until the length for which the fiber renewal with its capacity to bear the complete loading, therefore it must include the locally plasticized zone a $(\delta \geq a)$. According to the behavior laws and starting from the fractured zone, the natural broken fiber must pass by a locally plasticized zone $a$, then by transitory zone before reaching the perfectly elastic zone delimited by the length $\delta$ called ineffective under the effect of moisture and temperature (see Fig. 4). For different temperatures, the evolution of the ineffective length is studied as function of the number of broken fibers with a progressive variation of the moisture concentration from $0 \%$ to $100 \%$ for $\mathrm{T}=20^{\circ} \mathrm{C}, 90^{\circ} \mathrm{C}$ and $140{ }^{\circ} \mathrm{C}$, respectively.

For $T=20^{\circ} \mathrm{C}$, we obtain a superposition of all representative curves of the ineffective length. We can deduce that for low temperatures, the variation of moisture concentration have no effect on the ineffective length. With the increase in the temperature from $90{ }^{\circ} \mathrm{C}$ to $140{ }^{\circ} \mathrm{C}$, the sensitivity of the ineffective length for a variation of moisture concentration becomes increasingly important. For $T=140{ }^{\circ} \mathrm{C}$, the ineffective length for 40 broken fibers which is $1.32 \mathrm{~mm}$ for $C=0 \%$ becomes $1.15 \mathrm{~mm}$ for $C=100 \%$. We can deduce that the temperature is an important factor for the determination of the ineffective length passing from the carbon to hemp fiber, because it generates a degradation of the mechanical characteristics.

\subsection{Evolution of the stress concentrations}

The estimation of the length of locally plasticized zone, the ineffective length and the longitudinal displacement, allows the determination of the stress concentration factor. This factor is of great importance because it offers to us the ratio between the stress calculated at the fibers fracture point and the external applied stress. It will enable us to determine the stress concentration in the zone of the broken fibers, and to predict a probably fracture of neighboring adjacent fibers under the effect of moisture and temperature. It is well-known that the matrix is very sensitive to the variation of the temperature, and the interface adhesion between the hemp fibers and the matrix becomes very weak; thus the debonding phenomenon appears, and leading to the fiber fracture. When it appears a fiber fracture in the composite, an influence area was generated around the extremities of the broken fiber in which exists a stress concentration. In addition, the load can be transferred to a broken fiber with a shear stress at the fiber/matrix interface.

To illustrate the effect of the environmental conditions on the degradation of the ineffective area surrounded by the broken hemp fibers, in Figure 4, we present the evolution of the stress concentrations as function of the broken fibers for different variations of moisture concentrations and the humidity. 

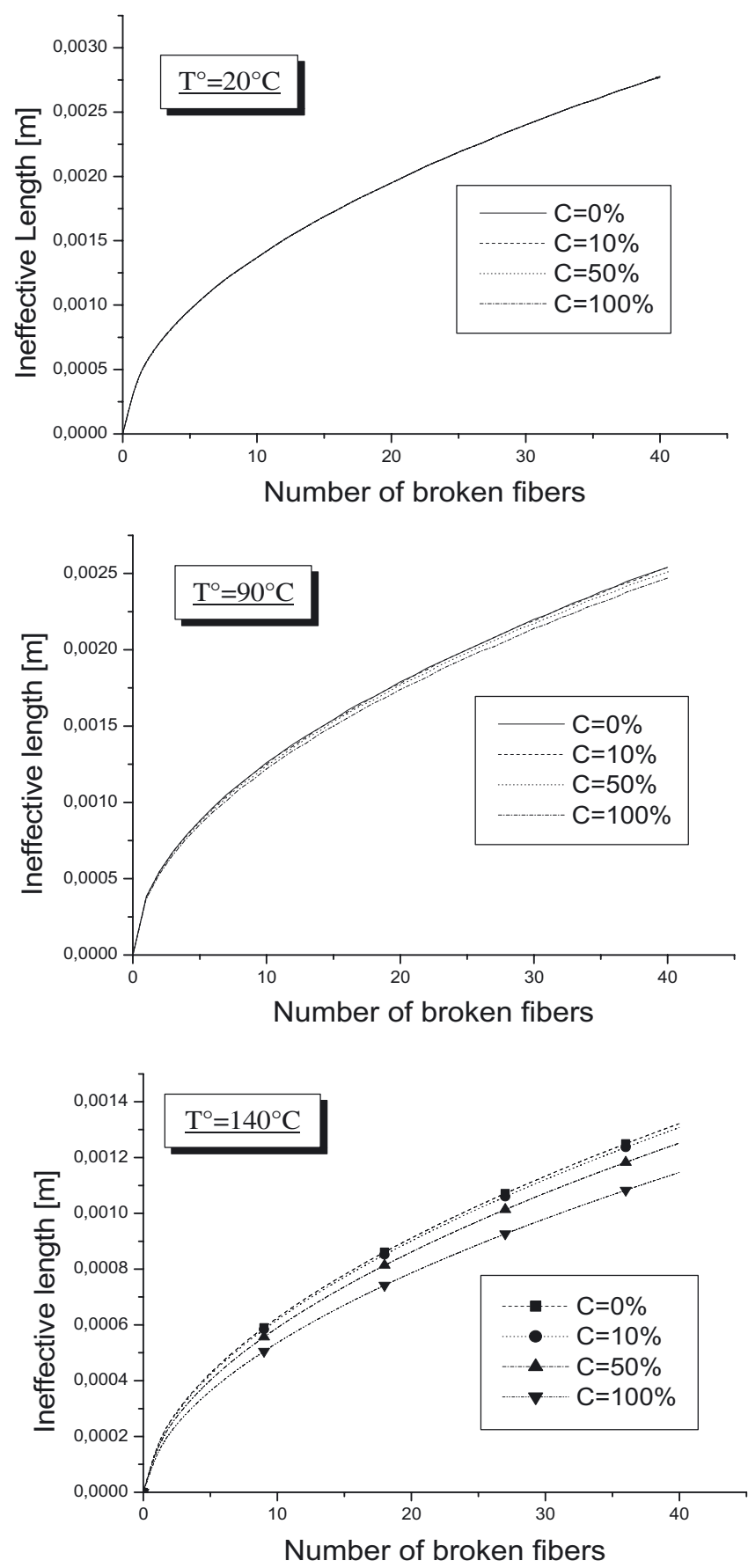

Fig. 4. Evolution of the ineffective length under various moisture concentrations for the temperatures $T=20^{\circ} \mathrm{C}, 90{ }^{\circ} \mathrm{C}$ and $T=140{ }^{\circ} \mathrm{C}\left(\sigma=0.5 \sigma_{0}\right)$.

The stress concentrations become more important with the increase of the number of broken fibers. Thereafter, this stress concentration becomes relatively stable if the number of fibers is very high. For the low temperatures, the effect of moisture concentration on the stress concentration is almost negligible as shown in Figure 5 for $T=20{ }^{\circ} \mathrm{C}$.

The effect of moisture concentration becomes increasingly important with the increase of the temperature from
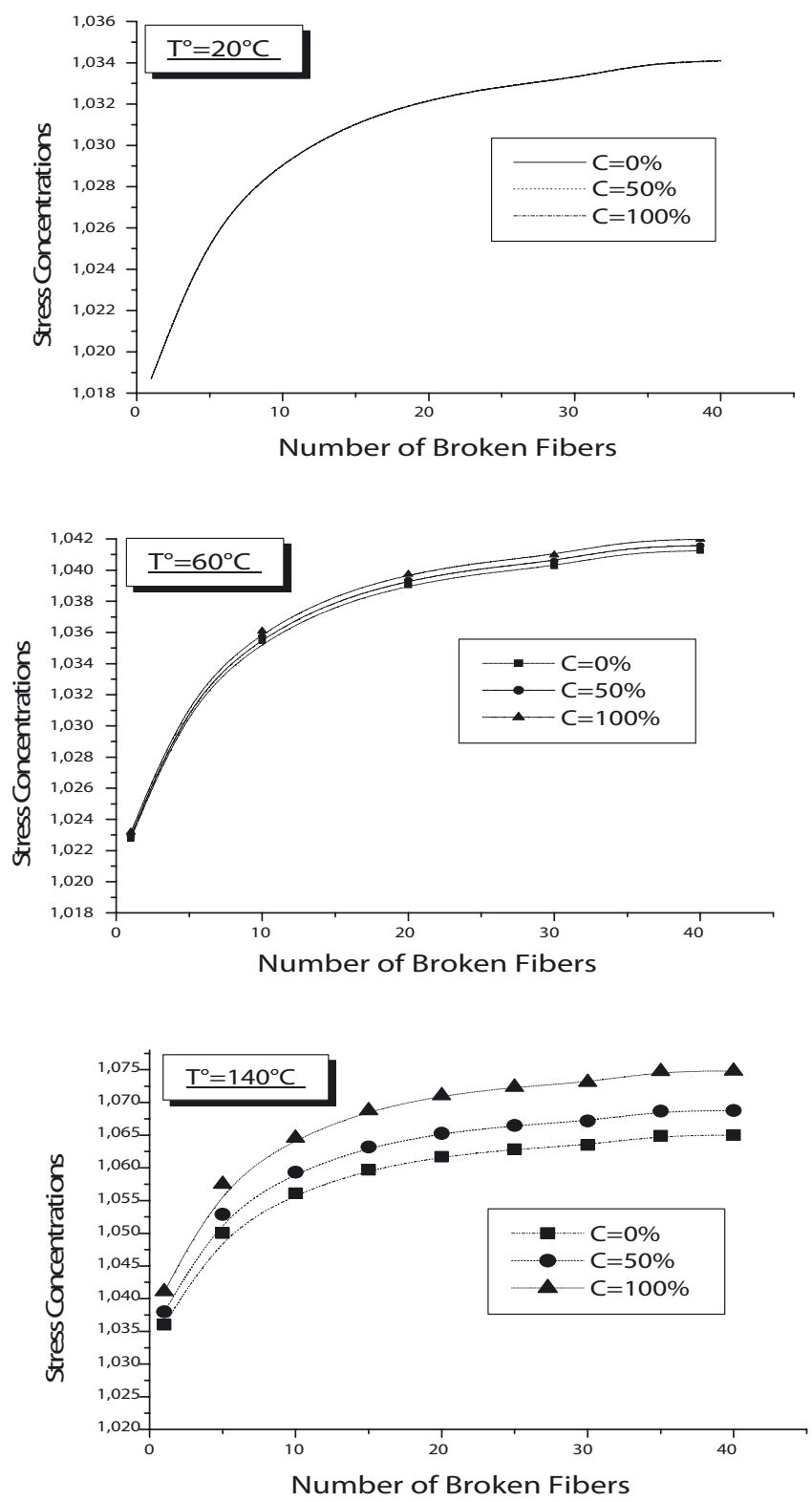

Fig. 5. Evolution of the stress concentration as function of broken fibers for a progressive variation of moisture concentration for $\sigma=0.5 \sigma_{0}$.

$90{ }^{\circ} \mathrm{C}$ to $140{ }^{\circ} \mathrm{C}$. For $T=140{ }^{\circ} \mathrm{C}$, a clear distinction appears between all the curves, for example; for 40 broken fibers, the stress concentration varies from 1065 for $C=0 \%$ to 1074 for $C=100 \%$. With the use of natural hemp fibers according to the above results, we can deduce that in the neighboring area of the intact fibers, the stress concentration is very high. But, when the number of broken fibers is very important, the stress concentration to the ineffective area is more important and the ineffective length is large. The neighboring area becomes less stiff, and the broken fibers react more easily and they can communicate together to cause a complete failure. 

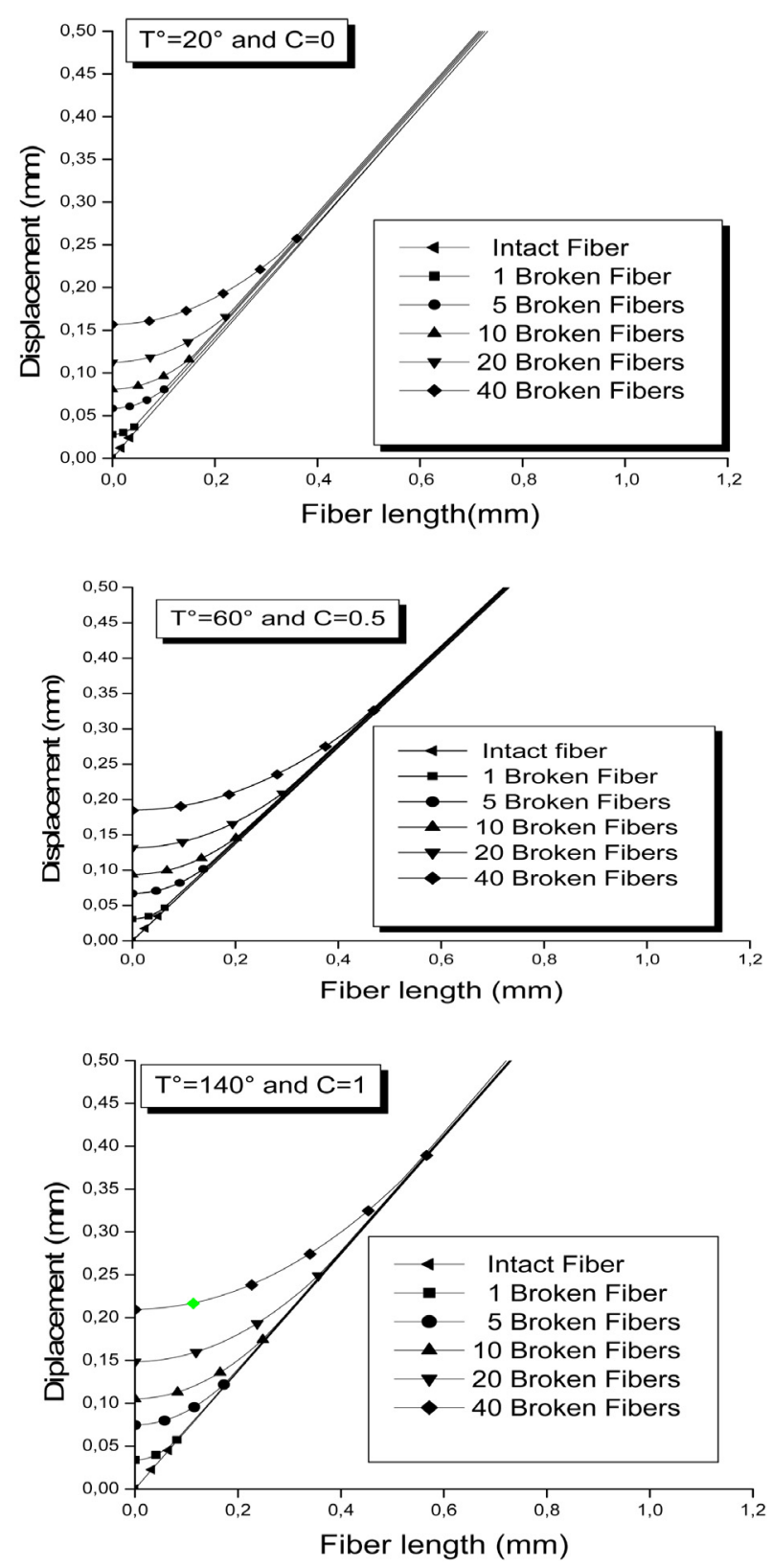

Fig. 6. Longitudinal displacement for broken and intact fibers surrounding the damaged zone for $\sigma=0.5 \sigma_{0}$.

\subsection{Evolution of longitudinal displacement at the failure fibers point}

According to the number of broken fibers, it is clear that the curves are distinguished one from the other (fibers failure point), then they have the same pace and they become lineal. We note that the longitudinal displacement for less than 5 broken fibers is less important while comparing by those of 40 broken fibers. Figure 6 , presents the effect of the environmental conditions on the evolutions of the longitudinal displacement in damaged zone.

By the variation in the temperature from $20{ }^{\circ} \mathrm{C}$ to $140{ }^{\circ} \mathrm{C}$ and moisture concentration from $0 \%$ to $100 \%$, the sensitivity of the model and the displacement on the level of damaged zone are less important, compared to the obtained results for stress concentration in the broken fibers zone. For example the longitudinal displacement for one broken fiber was less important passing from $0.036 \mathrm{~mm}$ for $T=20{ }^{\circ} \mathrm{C}$ and $C=0$ to $0.043 \mathrm{~mm}$ for the extreme conditions $\left(T=140{ }^{\circ} \mathrm{C}\right.$ and $\left.C=1\right)$, on the other hand the displacement for 40 broken fibers becomes more important in the vicinity of the damaged zone which passes from $0.1518 \mathrm{~mm}\left(T=20{ }^{\circ} \mathrm{C}\right.$ and $\left.C=0\right)$ to $0.2182 \mathrm{~mm}$ ( $T=140^{\circ} \mathrm{C}$ and $C=1$ ), for an external applied stress equal to 0.5 .

The relation between the longitudinal displacement and the length is lineal, if no fiber is broken. When the breaks appear, the curves take an exponential pace in the vicinity of the damaged zone, thereafter they become lineal, which reflects the no linearity phenomena in the damaged zone which is characterized by a local plasticity.

\section{Conclusions}

The purpose of the study was the analytical modeling of the behavior of hemp/epoxy unidirectional composite material under the effect of the environmental conditions. This behavior depends primarily on the arrangement, the orientation and the fiber fraction volume inside the matrix. The stress transfer from one fiber to another passes through the matrix in the presence of the shear stresses at the interfaces. The determination of the stress concentration factor makes it possible to have a clearer idea on the evolution of the fibers failure and the probability of damage of adjacent neighbor fibers. The vicinity of the failure zone is characterized by a locally plasticized zone which will become elastic while moving from it. Under the effect of a simultaneous variation of the temperature and the moisture concentration, the plastic zone and the ineffective area become widely according to the number of broken fibers and thereafter they become critical under the extreme environmental conditions, in addition it increases the longitudinal displacement in the plastic zone which leads to fast passage of the damage from a broken fiber to another, which permits us to be in the conformity with the micromechanical laws.

In other hand, the fabric hemp composites have advantages and disadvantages. Indeed, the hemp/epoxy composite, presents a great flexion stiffness. They are more resistant to the damage introduced by the flexion than the carbon fiber composites. The obtained results also showed that the fiber resistance and the Young modulus are relatively insensitive to the concentration of temperature and moisture, but the epoxy matrix is very influenced by the increase in the environmental conditions.

\section{References}

[1] C.M. Boyer, Composites unidirectionnels : modèles probabilistes de rupture et évaluation de la fiabilité, Thèse d'université, Blaise Pascal, 1997 
[2] P.M. Manne, S.W. Tsal, Design optimization of composite plates: Design criteria for strength, stiffness, and manufacturing complexity composite plates, J. Mater. Sci. 32 (1998) 544-571

[3] R.M. Christensen, Mechanics of composite materials, Krieger publishing company, Malabar, Florida, 1991

[4] B.W. Rosen, Tensile failure of fibrous composites, Am. Inst. Aeronaut. Astronaut J. 2 (1964) 1985-1991

[5] C. Zweben, Tensile failure of fiber composites, Am. Inst. Aeronaut. Astronaut J. 6 (1968) 2325-2331

[6] S.L. Phoenix, P. Schwartz, H. Robinson, Statistics for the Strength and Lifetime in Creep-Rupture of Model Carbon/Epoxy Composites, Composites Sci. Technol. 32 (1988) 81-120

[7] C.M. Landis, M.A. Mc Glockton, R.M. Mc Meeking, An Improved Shear Lag Model for Broken Fibers in Composite Materials, J. Composite Mater. 33 (1999) $667-680$

[8] C.M. Landis, R.M. Mc Meeking, Stress Concentrations in Composites with Interface Sliding, Matrix Stiffness, and Uneven Fiber Spacing Using Shear Lag Theory, Int. J. Solids Struct. 36 (1999) 4333-4361

[9] S.W. Case, G.P. Carman, J.J. Lesko, A.B. Fajardo, K.L. Reifsnider, Fiber Fracture in Unidirectional Composites, J. Composite Mater. 29 (1995) 208-228

[10] S.W. Case, K.L. Reifsnider, Micromechanical Analysis of Fiber Fracture in Unidirectional Composite Materials, Int. J. Solids Struct. 33 (1996) 3795-3812

[11] G.C. Foster, Tensile and Flexure Strength of Unidirectional Fiber-Reinforced Composites: Direct Numerical Simulations and Analytic Models, Master of Science in Engineering Mechanics, Virginia Polytechnic Institute and State University, 1998

[12] Z. Gao, K.L. Reifsnider, Micromechanics of Tensile Strength in Composite Systems, Composite Materials: Fatigue and Fracture, Fourth Volume, ASTM STP 1156 (1993) 453-470

[13] J. Gassan, A.K. Bledzki, Composites reinforced with cellulose based fibres, Progress Polymer Sci. 24 (1999) 221274

[14] S. Mishra, S.S. Tripathy, M. Misra, A.K. Mohanty, S.K. Nayak, Novel eco-friendly biocomposites; biofiber reinforced biodegradable polyester amide composites: fabrication and properties evaluation, J. Reinf. Plast. Comp. 21 (2002) 55-70
[15] A. O'Donnell, M.A. Dweib, R.P. Wool, Natural fibre composites with plant oil-based resin, Composites Sci. Technol 64 (2004) 1135-1145

[16] E.M. Pearce, Fibre Chemistry, Handbook of fibre science and technology, Menachem Lewin Eli M. Pearce, New York, Vol. IV, 1985 pp. 737-746

[17] K. Scrivener, H. Van Damme, Construction Materials: From Innovation to Conservation, MRS Bulletin, May 2004, pp. 308-312

[18] M.A. Khiat, Z. Sereir, A. Chateauneuf, Uncertainties of unidirectional composite strength under tensile loading and variation of environmental condition, Theor. Appl. Fracture Mech. 56 (2011) 169-179

[19] S. Roy, W. Xu, S. Patel, S. Case, Modeling of moisture diffusion in the presence of bi-axial damage in polymer matrix composite laminates, Int. J. Solids Struct. 38 (2001) 7627-7641

[20] Z. Sereir, N. Boualem, Effect of long term hygrothermal loadings and stacking sequences on the damage of hybrid composites, Theor. Appl. Fracture Mech. 47 (2007) 147163

[21] S.W. Tsai, Composite Design, Think Composites, 4th edition Dayton, 1988

[22] G. Camata, R. Corotis, E. Spacone, Simplified stochastic modeling and simulation of unidirectional fiber reinforced composites, Prob. Eng. Mech. 19 (2004) 33-40

[23] M.R. Raman, M.R. Wisnom, Three-dimensional Finite Element Analysis of the Stress Concentration at a Single Fiber Break, Composites Sci. Technol. 51 (1994) 517-524

[24] H.L. Cox, The elasticity and strength of paper and other fibrous materials, British J. Appl. Phys. 3 (1952) 72-79

[25] W.A. Curtin, N. Takeda, Tensile strength of fiberreinforced composites: II. Application to polymer matrix composites, J. Composite Mater. 32 (1998) 2060-2081

[26] C. Bonnafous, F. Lagattu, L. Chocinski, A. Abot, F. Dédaldéchamp, R. Lemoine, De la plante aux composites tissés à fibres de chanvre (Cannabis sativa), International Journées Scientifiques et Techniques AMAC MECAMAT Eco- Matériaux, Amiens, 13 Octobre 2008 